Thorax (1958), 13, 150.

\title{
THE PATHOLOGY AND BACTERIOLOGY OF RESECTED TUBERCULOUS LUNG LESIONS AFTER CHEMOTHERAPY
}

\author{
BY \\ D. J. COTTER, H. M. FOREMAN, AND R. M. E. SEAL \\ From Sully Hospital
}

(RECEIVED FOR PUBLICATION NOVEMBER 6, 1957)

The bacteriology of resected tuberculous lung lesions after varying amounts of chemotherapy has provided much important information on the efficacy of chemotherapy. As early as 1952 Medlar, Bernstein, and Steward (1952) and Beck and Yegian (1952) noted the effect of chemotherapy on tubercle bacilli. Few studies have been carried out where the bacteriology, histology, and chemotherapy have been correlated. Most reports have divided chemotherapy into groups according to duration only.

In this study of 218 patients, equal emphasis has been placed on the quality of chemotherapy and on its duration, and the resected lesions have been studied bacteriologically and histologically.

Factors other than chemotherapy may influence the progress of lesions, and in this study we have paid particular attention to one method of treating cavities, namely postural reduction (Thomas, 1955).

We hope to show that chemotherapy has only a slight influence on the consistence of caseous tissue, but has a marked tendency to collagenize the capsule and causes a dramatic reduction in culturable bacilli in the lesion, and that quality as well as duration of chemotherapy and size of the lesion also influence the bacteriology.

\section{Material AND Methods}

Clinical Material.-The group of 218 patients was representative of those found in any thoracic surgical unit. They were admitted between November, 1955, and November, 1956; ages varied from 19 months to 55 years, but $95 \%$ were over 15 years; the sex incidence was about equal. The duration of disease, always difficult to determine accurately, was taken as the time from diagnosis to operation. This period varied from 23 years to less than one year, but in the majority it was under three years. The radiological extent of the disease varied from the solitary lesion $1 \mathrm{~cm}$. and over in diameter to bilateral cavitated disease. In 22 patients, specimens from both lungs were examined.

Pre-operative Bacteriological State.Sputum specimens or gastric washings from all patients had at some time been cultured for tubercle bacilli, with a positive result in $75 \%$ of the patients. Of those who were positive, $64 \%$ had had sensitivity tests performed, and of those tested $32 \%$ had organisms showing some degree of resistance to one or more of the three drugs, streptomycin, p-amino-salicylic acid, and isoniazid. At the time of operation, however, cultures were positive in only six of the 218 patients, and of these four were resistant to one or more of the standard drugs.

Chemotherapy AdMinistered.-A review of the case histories of these patients showed that the chemotherapy they had received before admission to our hospitals varied greatly. Only a small percentage had what is now considered good chemotherapy.

We classed these chemotherapy régimes as follows :

$\begin{array}{llllll}\underset{\text { Good }}{\text { Indifferent }} & \ldots & \ldots & \ldots & \text { Group } & \mathbf{A} \\ \text { Bad .. } & \ldots & \ldots & \ldots & , & \text { B } \\ \text { Inadequate } & & \ldots & \ldots & , & \text { D }\end{array}$

Group A: Initial continuous chemotherapy in recognized combinations and dosage for a minimum period of eight months.

Group $C$ : Régimes which are known to be ineffective in preventing the emergence of resistant organisms, for example, single-drug therapy, inadequate dosage of drugs, twice-weekly streptomycin with another drug daily, to mention only three. Also included in this group were régimes including drugs to which the organisms were already resistant. 
Group D: Good régimes but of less than five months' duration.

Group $B$ : The remainder, consisting of acceptable régimes continuously for five to eight months, or for a total of more than five months with interruptions. Comparison of those with intervals in therapy of more than three months and those with intervals of less than three months (but more than one month) did not reveal any significant difference in the results in the two groups, and we therefore have not subdivided according to the duration of the interruptions.

All except two of these 218 patients received isoniazid at some stage of treatment.

The distribution of patients in these four groups was as follows:

\begin{tabular}{|c|c|c|c|c|}
\hline Group & \multicolumn{2}{|c|}{ Classification } & $\%$ & $\begin{array}{l}\text { Duration of Treatment } \\
\text { (Average in Months) }\end{array}$ \\
\hline $\begin{array}{l}\mathbf{A} \\
\mathbf{B} \\
\mathbf{C} \\
\mathbf{D}\end{array}$ & $\begin{array}{l}\text { Good } \\
\text { Indifferent } \\
\text { Bad } \\
\text { Inadequate }\end{array}$ & $\begin{array}{l}\ddot{ } \\
\cdots \\
\cdots\end{array}$ & $\begin{array}{l}19 \\
35 \\
35 \\
11\end{array}$ & $\begin{array}{c}12 \frac{1}{2} \\
8 \frac{1}{2} \\
10 \\
3 \frac{1}{2}\end{array}$ \\
\hline
\end{tabular}

Ancillary Treatment.-At hospitals where the patients in this series were finally assessed and treated before surgery, strict posture is used for the reduction of cavities. The patient lies on the affected side in a plaster cast to maintain the cavity in a dependent position relative to its draining bronchus throughout the day and during sleep (Thomas, 1955).

Laboratory Material and Methods.-Two hundred and forty specimens were examined from these 218 patients studied, 22 of whom provided material from both lungs. Caseous material was always sampled aseptically from a cavity when felt, otherwise from the largest caseous lesion, and the lesion measured. The material was divided. One portion was retained in our laboratory for treatment with an equal volume of $3 \%$ sulphuric acid for $5 \mathrm{~min}$. before dilution. After dilution with $7 \mathrm{vol}$. of distilled water, the specimen was centrifuged at 3,000 r.p.m. for 15 min.

For direct examination a film of the deposit was stained by the Ziehl-Neelsen technique, and scrutinized using a 1/7 oil immersion lens and a green filter. On 50 occasions films were also made by directly smearing the caseous tissue. No additional yield of positives resulted.

The second portion was examined by Dr. J. Marks at the Central Tuberculosis Laboratory, Cardiff. With the first 200 specimens, the untreated material was inoculated into guinea-pigs after grinding with sterile saline in a Griffith's

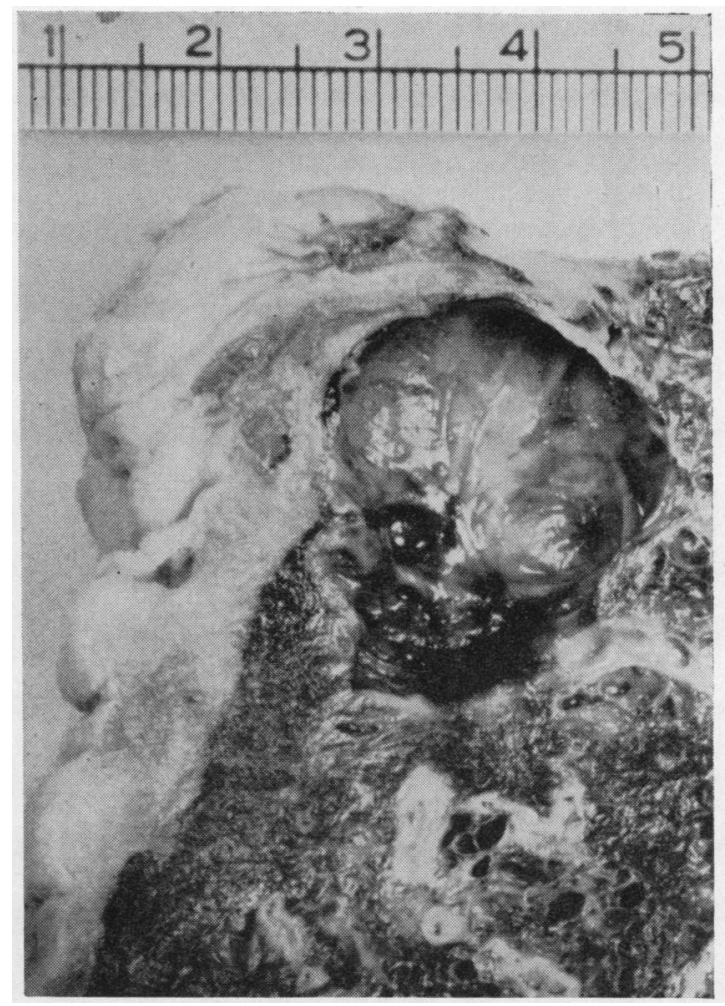

FIG. 1.-Smooth-walled apical cavity which on microscopy was fibrous lined but with occasional areas of epithelioid cells and giant cells.

tube. Cultures were made by treating the material with $5 \%$ sulphuric acid for $10 \mathrm{~min}$. before diluting, centrifuging and inoculating Löwenstein-Jensen and Kirschner's media with the deposit. On 100 occasions Löwenstein-Jensen slopes were inoculated with untreated material, and no increased yield of positives resulted.

Cultures were incubated for three months, and, when positive, sensitivity tests were performed by Dr. Marks using his published technique (Marks, 1956). Catalase tests were performed on only a few occasions.

Morbid ANATOMy.-After formalin fixation the specimen was carefully examined. Open cavities were classed as "chronic open" if lined by ragged necrotic tissue. If the lining was glistening (Fig. 1), they were termed "smooth walled." Cavities were still classed as "smooth walled" if later microscopy revealed some areas of tuberculous granulation tissue with necrosis in addition to the usual collagenous wall. As only caseous lesions were sampled, and few smooth-walled cavities have any obvious necrotic contents, only two were 
examined bacteriologically. Similarly, stellate scars (Fig. 2), though recorded, were not examined bacteriologically.

At the time when the caseous lesion was bacteriologically sampled, it was assessed as calcified when this was obvious to the scalpel, solid when it had to be dug out, and pultaceous when the material could be expressed with ease. If the lesion represented a blocked cavity (Fig. 3), it was classified as such. To distinguish between a pultaceous lesion which has never been a cavity and a blocked cavity is difficult, and it is recognized that false assessments may have been made.

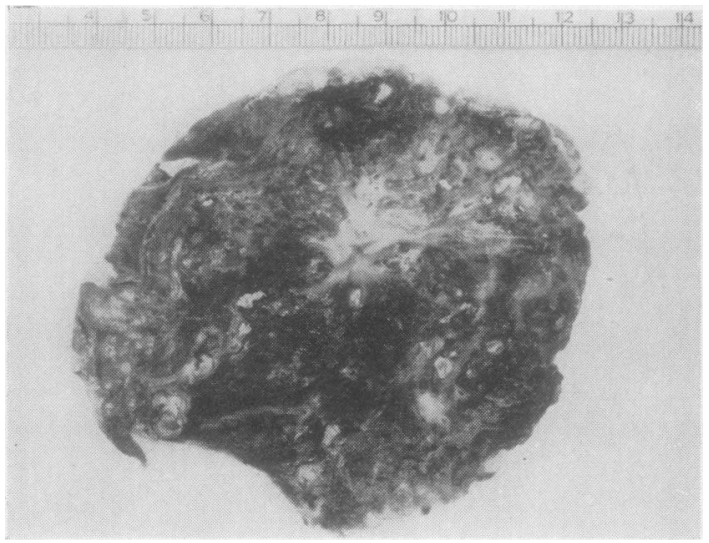

Fig. 2.-A typical "stellate scar" and a few caseous foci.

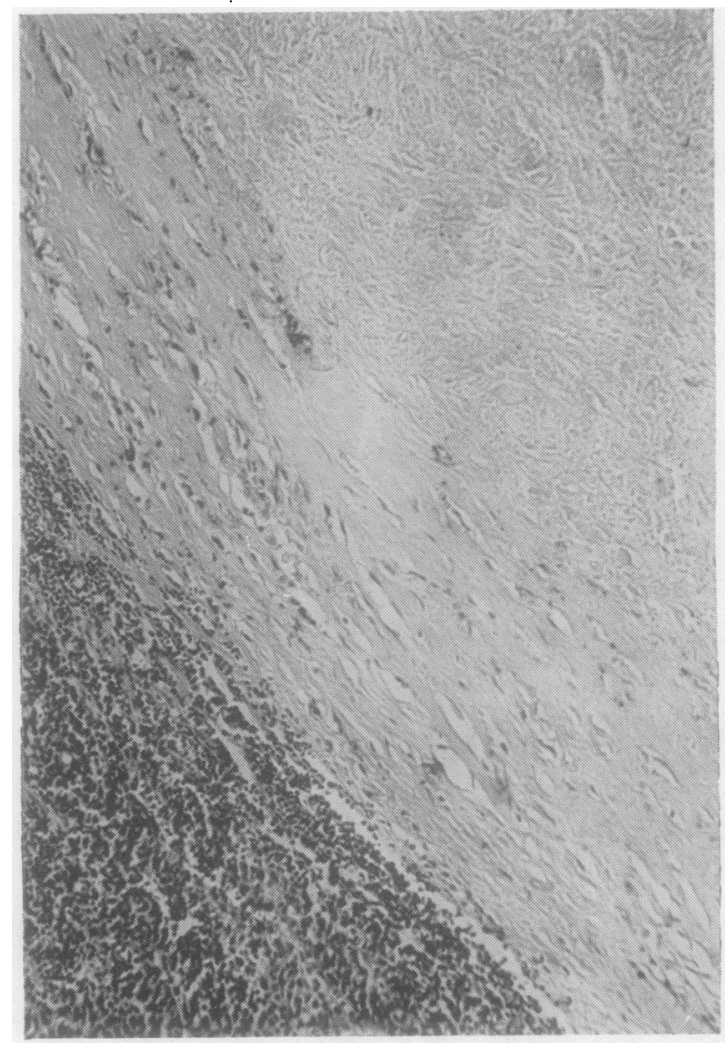

Fig. 4.-Histology of capsule of caseous focus, Group I. The capsule is collagenous, without an inner zone of epithelioid or giant cells. Haematoxylin and eosin, $\times 120$.

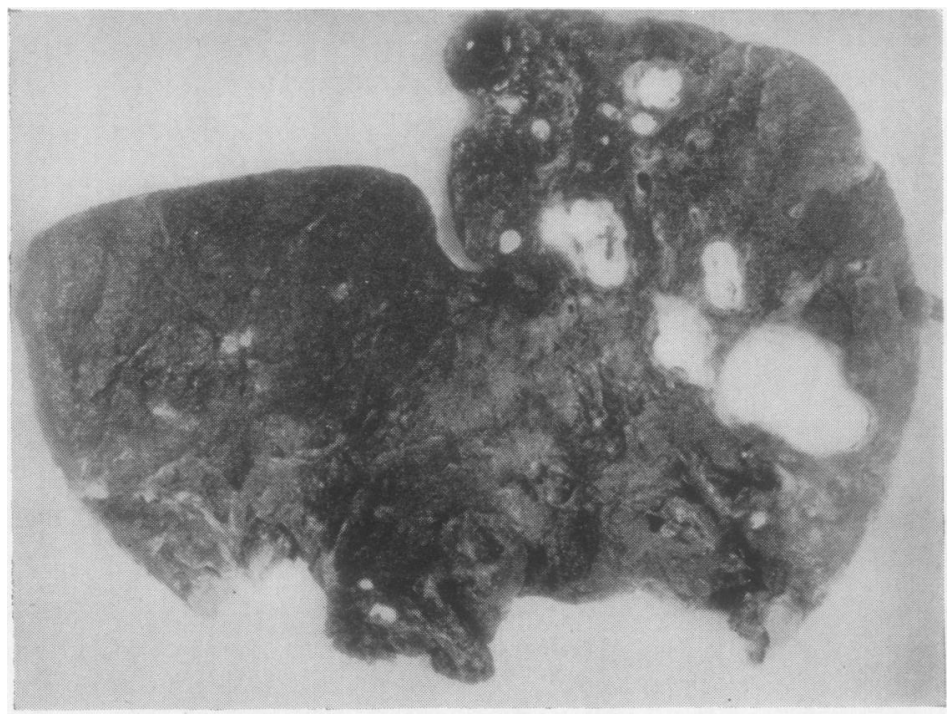

FiG. 3.-A “ blocked cavity" (bottom right) contrasting with the dust mottled solid caseous lesions. 


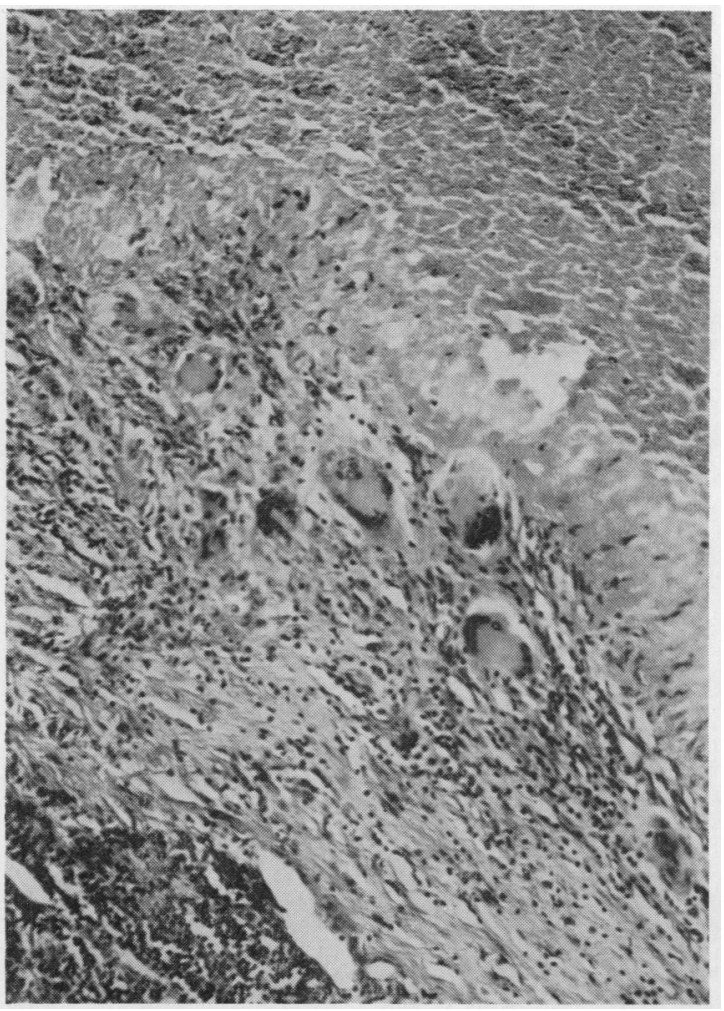

Fig. 5.-Histology of capsule of caseous focus, Group II. The capsule is collagenous with a cellular zone of epithelioid cells between the collagen and the caseous material. Haematoxylin and $\operatorname{cosin}, \times 120$.

A block of tissue for histology was invariably taken from the lesion taken for bacteriology. The histology of the capsule fell into three groups :

Group I: when a collagenous capsule with or without an exterior lymphocytic zone limited the caseous material (Fig. 4).

Group II: when a cellular zone of epithelioid cells with or without giant cells was interposed between the collagen and the caseation (Fig. 5).

Group 11I : when a cellular zone only, without a collagenous capsule, limited the caseation (Fig. 6).

\section{RESUlts}

Overall Bacteriological Findings.-In $65 \%$ of the lesions acid-fast bacilli could be seen on direct examination, but only $12 \%$ were positive on culture. Of the 200 guinea-pigs inoculated, $10.7 \%$ of those escaping intercurrent infection were positive. Only on two occasions was the guineapig test positive and the cultures negative, but on four occasions the cultures were positive and the guinea-pig test negative. In these four cases the bacilli were resistant to isoniazid, and animal inoculation with the culture showed attenuation of virulence.

The higher incidence of visible than of culturable bacilli in lesions is a well-recognized finding after chemotherapy, as is the isolation of some organisms of attenuated virulence.

The overall proportions of $65 \%$ of lesions with visible and $12 \%$ with culturable bacilli are similar to those reported in many of the published series. Few of these series are comparable, however, in the material sampled, the methods employed, or the chemotherapy administered. Stewart, Turnbull, and Macgregor (1956), in 194 caseous foci from patients with a negative sputum preoperatively and treated with drugs to which the organisms were known to be sensitive, found $75 \%$ positive on direct examination and $9 \%$ positive on culture. In contrast Hurford and Valentine (1957) in a series of 50 patients after varying periods of continuous chemotherapy found $80 \%$ with a positive smear and $38 \%$ positive on cul-

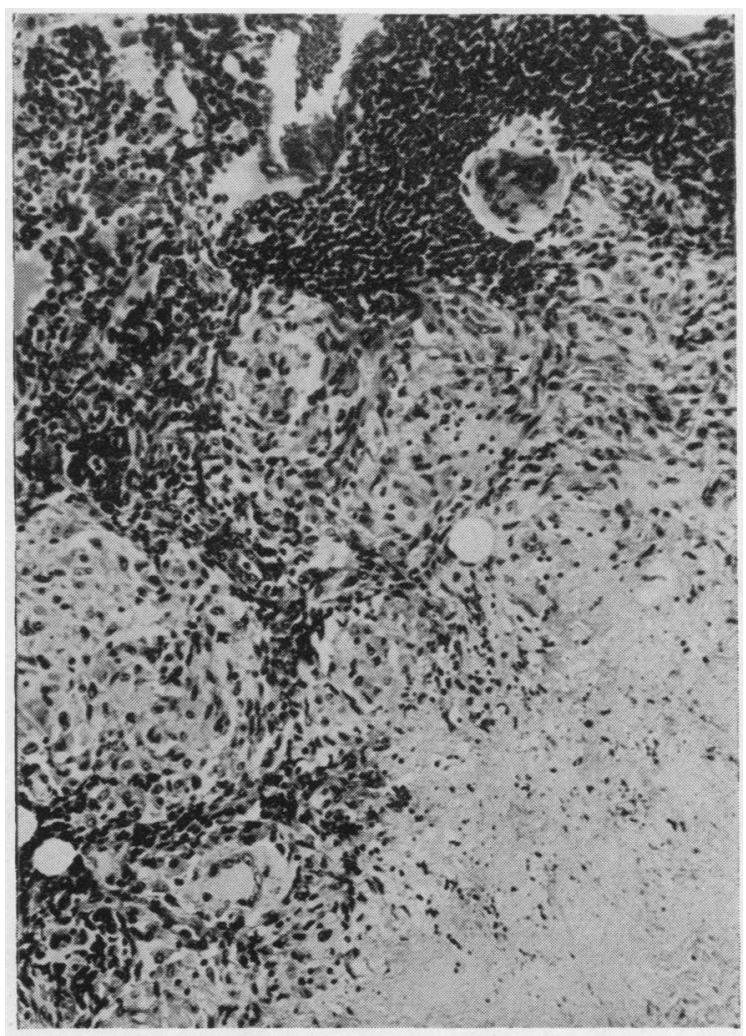

Fig. 6.-Histology of capsule of caseous focus, Group III. The capsule is cellular, without a collagenous zone. Haematoxylin and cosin. $\times 120$. 
ture or guinea-pig inoculation. This is a significantly higher percentage of viable organisms, and is difficult to explain as they found no increased yield of positives with prolonged incubation, and their inoculation of six Löwenstein-Jensen slopes, instead of two as in our series, is unlikely to have yielded the extra positives. It would appear that the material and chemotherapy in the two series differed in some important respects.

\section{The Influence of the Various Types of Chemotherapy on Lesion Consistence, Histology, and BaCteriology}

Chemotherapy and Consistence.-There were 12 calcified, 142 solid, and 86 pultaceous lesions. The influence of chemotherapy on consistence was found to be slight as shown in Fig. 7. There were slightly more calcified and slightly fewer pultaceous lesions in those patients who had received "A" type chemotherapy. As was to be expected when these patients were also considered according to the duration of their disease, it was found that significantly more patients with duration of disease of more than three years had solid as opposed to pultaceous lesions in the " $\mathrm{D}$ " chemotherapy group. In patients receiving " A " type chemotherapy the influence of duration of disease was negligible, suggesting that "A" type chemotherapy caused an alteration in consistence which outweighed the influence of the duration of disease.

As there were only 12 calcified lesions out of a total of 240 it is clear that chemotherapy has no important influence towards calcification, but the findings suggest that with longer periods of chemotherapy lesions tend to solidify.

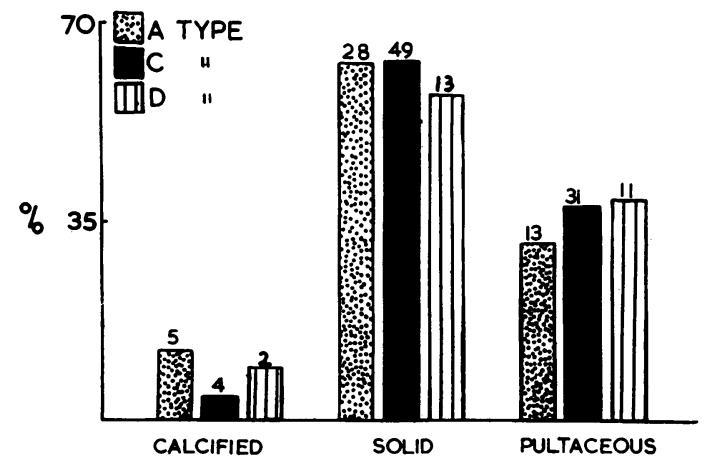

FIG. 7.-Effect of types of chemotherapy on consistence of caseous lesions. The height of each column indicates the percentage of patients receiving the type of chemotherapy indicated by the shading in whom the caseous lesions were calcified, solid, or pultaceous. For definition of types of chemotherapy, see text. In this and subsequent diagrams, the number of patients in the group indicated by each column is given at its head.

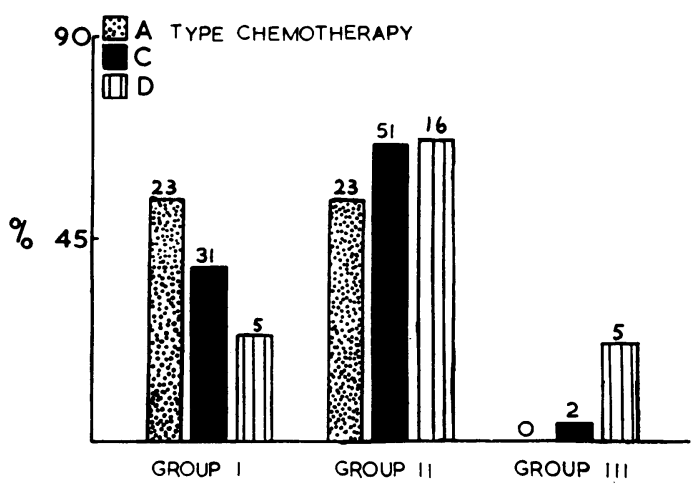

FIG. 8.-Effect of types of chemotherapy on the histology of the aapsule of caseous lesions. The height of each column indicates the percentage of patients receiving the type of chemotherapy indicated by the shading in whom the lesions were limited by Groups I, II, and III histology. See text and preceding figures.

Chemotherapy and Histology of Capsule.There were 87 lesions limited by collagen only (Group I) ; 144 limited by collagen and a cellular zone (Group II); and only nine limited by a purely cellular zone (Group III).

When the chemotherapy given was correlated with the histology of the capsule of the lesions (Fig. 8), it was found that all those who had "A" type chemotherapy possessed collagenous capsules (Groups I and II). Those who had cellular capsules only (Group III) had received either "D" or "C" chemotherapy.

When the duration of disease was also considered, it was found to be related to the type of capsule. Duration of disease appeared to influence the distribution of lesions with Group II and Group III histology in patients receiving the shorter "D" chemotherapy, as no lesions were found limited by an entirely cellular capsule when the disease was of more than three years' duration. The influence of duration of disease was also seen after " A" type chemotherapy, but it was less.

As patients in each group were comparable apart from the chemotherapy received, it would appear that efficient chemotherapy has a definite influence on the histology of the capsule, effecting a change toward collagenous encapsulation, and accelerates the change that can occur naturally in favourable circumstances.

As the great majority of our patients received isoniazid, we could not determine whether isoniazid had any particular influence on histo$\log y$.

CHEMOTHERAPY AND BACTERIOLOGY.-The effect of chemotherapy on the bacteriology was considerable, and the difference between the four 


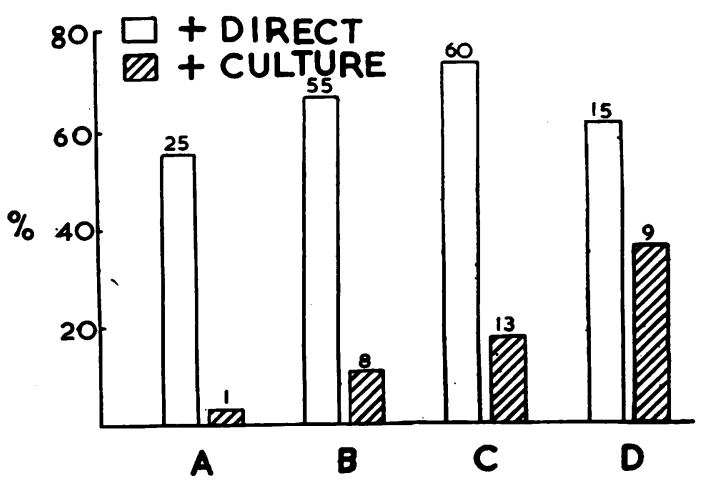

FIG. 9.-Results of bacteriological examination of caseous lesions after the types A, B, and C and D chemotherapy (for definition see text). The height of the unshaded columns represents the percentage positive on direct examination and the shaded the percentage positive on culture.

main types was clear-cut (Fig. 9). There is a marked reduction in the percentage of lesions positive on culture with improving chemotherapy, and of the 41 patients receiving " $A$ " chemotherapy only one was positive on culture. With the exception of " $D$ " chemotherapy there is also a reduction in the percentage positive on direct examination with improving chemotherapy, even though after " A" chemotherapy over a half of the lesions still contained acid-fast bacilli.

The only lesion positive on culture in the " $A$ " chemotherapy group was from a patient treated with three times weekly streptomycin and P.A.S. daily $\left(S_{3} P\right)$. This régime was included as generally acceptable, but this single exception supports the opinion of Stewart and others (1956) that it is not an effective combination. It is clear that quality of chemotherapy is as important as its duration. " C" type chemotherapy, which had an average duration approaching that of "A" type, and of longer average duration than " $B$ " type, yielded a higher percentage positive on film and culture.

As the majority of lesions sampled were solid and non-cavitated, and bacteriological results in these varied with the chemotherapy, we cannot agree with Canetti, Israel, Hertzog, Daumet, and Toty (1954) that chemotherapy has little influence on solid lesions as opposed to cavities.

As was to be expected, the great majority of positive lesions with resistant organisms occurred in patients after " $\mathrm{C}$ " type chemotherapy, there being only one example of resistant organisms in lesions after "A" chemotherapy, and that the $\mathrm{S}_{3} \mathrm{P}$ régime, and one example after " $\mathrm{B}$ " type chemotherapy.

Of 34 patients with resistant organisms present at some time in sputum before operation, $37 \%$ had lesions positive on culture compared with $7 \%$ of those with fully sensitive organisms. The great majority of these resistant organisms followed " $C$ " chemotherapy.

These findings emphasize that development of resistance is an important factor in the failure of chemotherapy to make lesions culture negative, and that improper régimes are associated with the persistence of resistant organisms. Our findings reinforce the views of Stewart and others (1956).

Abnormal morphology of the tubercle bacilli (Fig. 10) was seen in $40 \%$ of the positives. Bacilli frequently showed clubbed ends and an appearance simulating spores, which were sometimes central, terminal, or subterminal. There was no definite relation between direct positives showing abnormal morphology and negative cultures, however. These morphologically abnormal bacilli have been noted previously (Hurford and Valentine, 1957).

The presence of acid-fast bacilli in apparently sterile caseous material has occasioned much speculation. D'Esopo, Bernstein, Decker, Raleigh, and Steenken (1953) and Stewart and others (1956) consider them dead, whilst Dubos (1953), Ware,

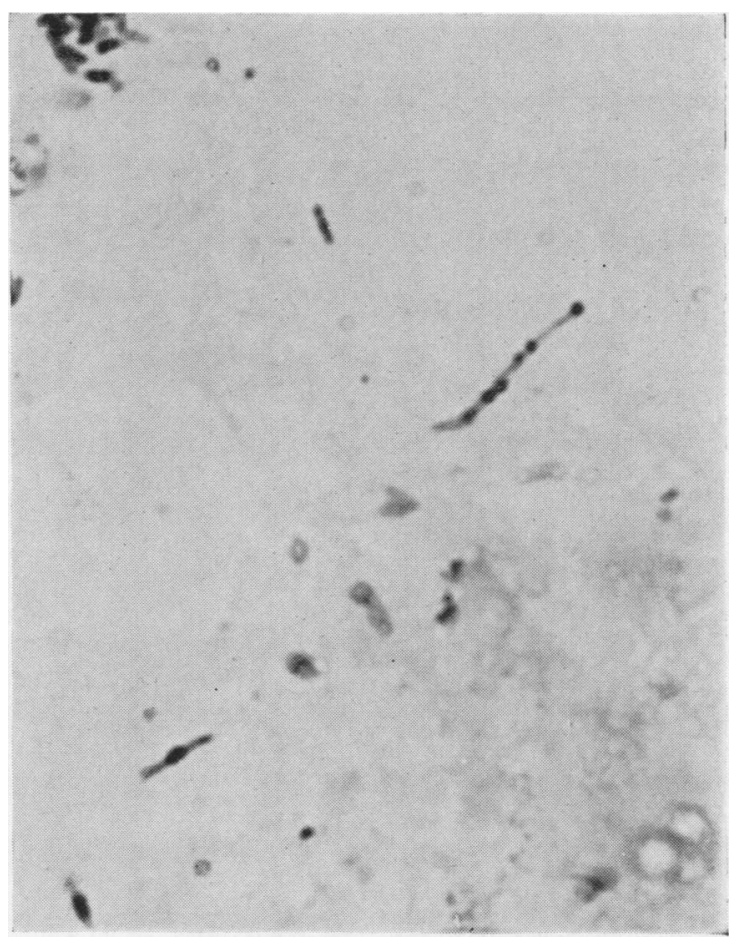

FIG. 10.-Abnormal morphology of tubercle bacilli from a caseous lesion after chemotherapy. Ziehl-Neelsen stain, $\times 2,300$. 
Stauss, Dillon, and Tempel (1956), and Hurford and Valentine (1957) are doubtful.

We feel that, though doubt remains concerning the non-viability of the visible bacilli, sufficient bacteriological evidence is accumulating to justify a conservative policy in patients who are known to have received prolonged good chemotherapy. Our results suggest this certainly holds for residual solid lesions, and though the numbers of residual cavities sampled was small, a conservative policy may prove to be justified in residual cavitary disease also.

Size of Lesion in Relation to Bacteriology. -Fig. 11 shows that the percentage of positives on direct examination increases in proportion to the size of the lesion. Analysis of each of the chemotherapy groups in turn shows a similar pattern. Culture results in relation to size of lesion also showed a higher percentage of positives as the lesion size increased. Analysis of the individual chemotherapy groups " B," " $\mathrm{C}$," and "D" showed the same trend, but with "A" chemotherapy only one positive culture was found, whatever the size of the lesion.

Difference in bacterial content of lesions according to their size has been noted before; Canetti and others (1954) found increased positives with larger lesions. Our findings in 240 measured lesions emphasize this, and the results after " $A$ " chemotherapy support the view of Stewart and others (1956) that duration of chemotherapy is very important, and that the largest can be made negative on culture after good chemotherapy.

The smaller number of A.F.B. and positive cultures from smaller lesions is not unexpected when we consider the good results achieved after resection, when small lesions are left in situ. This concept of the importance of size has also influenced some clinicians in the need for resection in

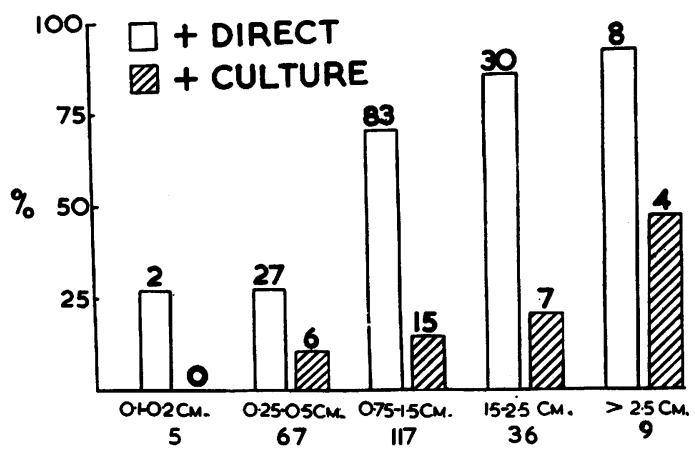

Fig. 11.-Bacteriology of caseous esions of different size.

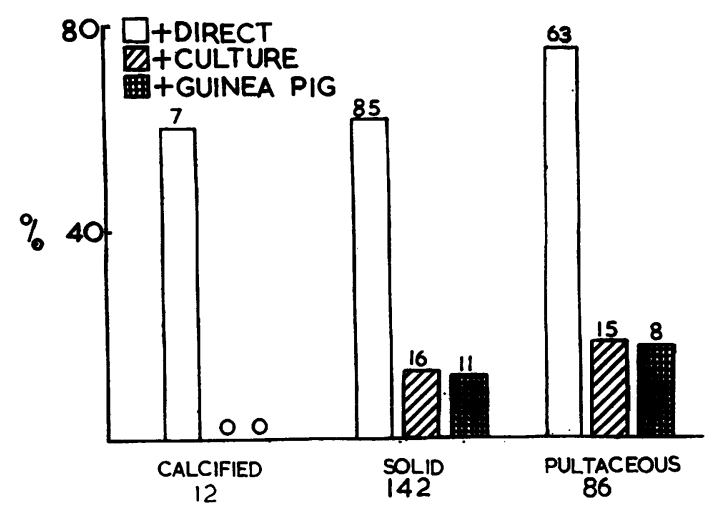

Fig. 12.-Bacteriology of lesions of different consistence. The heights of unshaded, hatched, and double hatched columns represent the percentage positive on direct examination, culture, and guinea-pig inoculation respectively. The numbers at the head of the column represent the number constituting the group. For guinea-pig inoculation the percentage positive was related to the number inoculated and escaping intercurrent infection.

their patients, and in our material $16 \%$ of lesions over $0.75 \mathrm{~cm}$. were culture positive compared with $8 \%$ of lesions smaller than $0.75 \mathrm{~cm}$.

An implication of our findings, we think, is that more attention might be paid to lesion size in decisions about treatment. Is it possible that periods of chemotherapy shorter than eight to 12 months will suffice for the smaller lesions? If the bacteria remaining in caseous lesions were not dead (and $88 \%$ of lesions over $1.5 \mathrm{~cm}$. contained acid-fast bacilli after " A" type chemotherapy), the indication for resection would be the greater the larger the lesion.

CONSISTENCE AND BACTERIOLOGY.-There was a correlation between the consistence and bacteriology (Fig. 12), the pultaceous lesions having most direct, culture, and guinea-pig positives. It is interesting to note that though seven of the 12 calcified lesions were positive on direct examination, none was positive on culture. This finding is in direct contrast to that of Canetti (1955) in lesions calcifying without chemotherapy. He could not see tubercle bacilli in these lesions but found $50 \%$ positive on guinea-pig inoculation.

Our findings suggest that, from a purely bacteriological standpoint, patients presenting with calcified lesions would benefit from chemotherapy.

HistologY AND BACTERIOLOGY.-There was a relationship between the histology of the capsule and the bacteriology of the lesion (Fig. 13). Lesions limited by a cellular zone only (Group III) were all culture positive, whilst those limited by collagen only (Group I) were all culture negative, though $52 \%$ were positive on direct examina- 


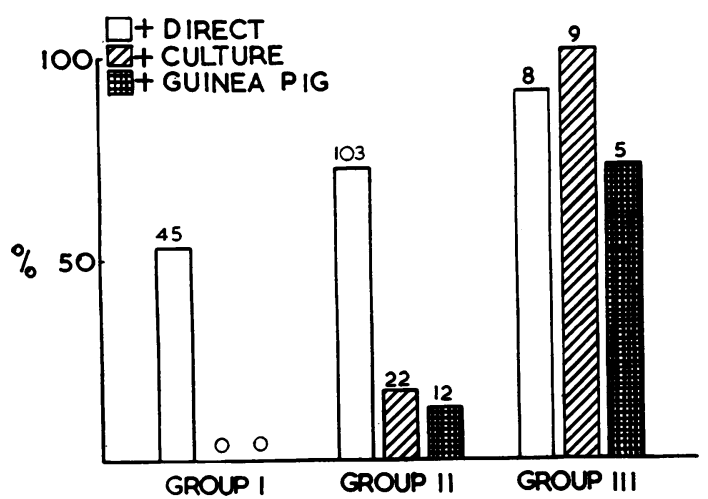

Fig. 13.-Bacteriology of caseous lesions with different types of histology of the capsule. Again the percentage of guinea-pig positives was related to those inoculated and escaping intercurrent infection.

tion. Lesions limited by endothelioid cells, giant cells, and an outer collagenous capsule (Group II) were negative on culture after " $A$ " chemotherapy. It would appear unjustifiable to assume that the presence of cellular areas around solid caseous lesions and in cavity walls indicates activity.

It is interesting that the caseous pulmonary and lymph node components of the primary lesions studied in great histological detail by Sweany (1941) took many years to become purely collagenencapsulated. Sweany infers that the natural encapsulation is a slow process, which takes about 10 years, and under natural conditions it was only after this period that he failed to recover tubercle bacilli.

It would appear, therefore, that proper chemotherapy given for long enough greatly hastens the histological and bacteriological changes occurring naturally under favourable conditions, achieving in one year what in the unaided host takes 10 years or more.

Cavity State.-Of 86 cavities, including blocked cavities and stellate scars resulting from cavity healing in the resected specimens, the distribution of types was as follows:

$\begin{array}{llllr}\text { Chronic open } & \ldots & \ldots & \ldots & 18 \\ \text { Smooth walled } & \ldots & \ldots & \ldots & 8 \\ \text { Blocked } . & \ldots & \ldots & \ldots & 50 \\ \text { Stellate scar } & \ldots & \ldots & \ldots & 10\end{array}$

In these 218 patients, only 26 open cavities were found in the resected specimens; this is a lower incidence of open cavitation than might be expected even with uniformly good chemotherapy (Livings, 1957), although in this series only $19 \%$ had such good treatment.
All cavities seen, blocked and open, were measured, and only $8 \%$ were larger than $2.5 \mathrm{~cm}$.; $32 \%$ measured between $1.5 \mathrm{~cm}$. and $2.5 \mathrm{~cm}$. ; $60 \%$ were less than $1.5 \mathrm{~cm}$. in diameter. This was considered to be a very satisfactory end result, and the factors to which it could be attributed were sought.

Ninety-three patients shown radiologically to have had cavities and treated by chemotherapy and posture were considered. It was found that in $80 \%$ of these cavity closure had been achieved. In $37 \%$ blocked cavities were recognized; $3 \%$ had stellate scars, and in the remaining $40 \%$ solid caseous lesions only were reported. This figure of $40 \%$ may seem unduly high, but, as pointed out, the differentiation between blocked cavities and other solid tuberculous lesions is often difficult. Nevertheless, it does not alter in any way the overall figure of $80 \%$ of patients with cavities having achieved a satisfactory end result.

The factors known to affect cavity closure, and which were common to these 93 patients, were chemotherapy and posture. We therefore correlated chemotherapy with cavity closure (Fig. 14). It will be seen that, in the group receiving the best type of chemotherapy, there is the lowest percentage of cavity closure ; it is noteworthy, however, that many of these open cavities were smooth walled. The other chemotherapy groups differ but little. The satisfactory closure rate, in this series, was not related to the quality of the chemotherapy, and we therefore think that posture has contributed substantially.

Perhaps of greater importance than its physical state is the bacteriological state of a cavity. Only 11 of the 26 open cavities were noted before fixation and studied bacteriologically, and though the

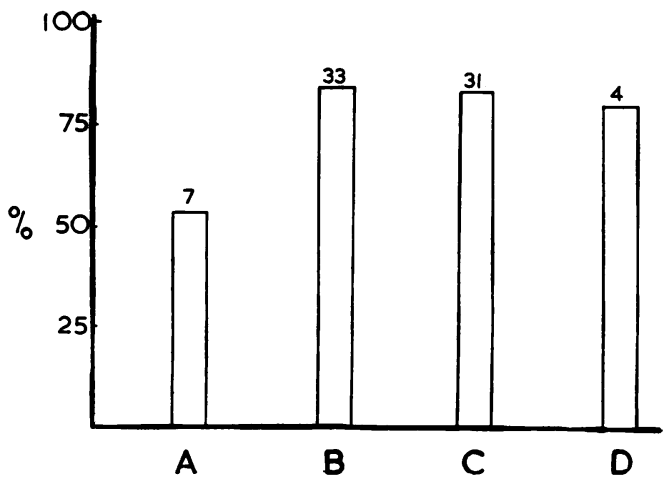

FIG. 14.-Cavity closure after differing types of chemotherapy. The height of the columns represents the percentage of patients with cavities, in which subsequent examination of resected specimens showed cavity closure. 
numbers are small the results in relation to chemotherapy are significant. There were no positive cultures from five cavities after "A" chemotherapy, one positive out of three after " $B$," one out of two after " $C$," and the one cavity examined after " $D$ " was positive.

It would appear that in this series posture has contributed considerably to the high cavity closure rate, and chemotherapy has been responsible for the "sterilization" of cavities.

Because the number of open cavities examined bacteriologically was so small compared with the number of blocked cavities examined, we can give no indication whether cavity reduction aided by posture is accompanied by any favourable effect on the bacteriology. It was clear, however, that blocked cavities were more frequently positive than "solid" lesions, suggesting that the former are potentially more dangerous. Postural reduction appears to be a valuable ancillary method for reducing cavity size and aiding closure, but its effect on the bacteriology remains uncertain.

\section{SUMmaRY AND CONClUSIONS}

Two hundred and forty resection specimens from 218 patients treated with anti-tuberculosis drugs were examined bacteriologically and in $65 \%$ acid-fast bacilli were seen, but tubercle bacilli were grown in $12 \%$ only.

Chemotherapy had a slight influence on the consistence of the lesions, tending to induce solidification, and a definite influence on the histology of the capsule, speeding the tendency to fibrous encapsulation. After good chemotherapy no positive cultures were found, after indifferent $12 \%$, after bad régimes of long duration $18 \%$, and after good chemotherapy of short duration $35 \%$ were positive on culture.

It was found that the development of bacteriological resistance was an important factor in the persisting positivity of resected lesions, and that the development of resistance was associated with bad chemotherapy.

The size of the lesions influenced the bacteriology, larger lesions being more frequently positive on direct examination and culture. After good chemotherapy, however, even the largest lesions failed to yield positive cultures.

In 93 patients known to have cavitated disease, cavity closure was achieved in $80 \%$ : this good result was largely attributed to postural reduction rather than to the chemotherapy administered.

We thank our colleagues, Mr. H. R. S. Harley, Mr. T. H. L. Rosser, Mr. D. M. E. Thomas, and Dr. L. R. West, for their helpful co-operation, and Dr. Emrys Jones for access to clinical records of patients treated in Glan Ely Hospital.

We are indebted to Dr. J. Marks for much of the bacteriology, and to Professor J. Gough for helpful criticism and advice.

We are grateful to Mr. D. Pugh, Miss J. Vincent, Mr. J. Hopkins, and Miss A. Waller for undertaking extra technical work, and Miss P. Edwards and Miss P. Morse for secretarial help.

\section{REFERENCES}

Beck, F., and Yegian, D. (1952). Amer. Rev. Tuberc., 66, 44.

Canetti, G. (1955). The Tubercle Bacillus in the Pulmonary Lesion

of Man. Springer, New York.
- Israel, R., Hertzog, P., Daumet, P., and Toty, L. (1954). Poumon, 10, 465.

D'Esopo, N., Bernstein, S., Decker, A. M., Raleigh, J. W., and Steenken, W., Jr. (1953). Transactions of the 12 th Conference on Chemotherapy of Tuberculosis, U.S. Veterans' Administration, Army and Navy, p. 229.

Dubos, R. J. (1953). Amer. Rev. Tuberc., 67, 874

Hurford, J. V., and Valentine, W. H. (1957). Tubercle (Lond.), 38, 194.

Livings, Dorothy G. (1957). Transactions of the 16th Conference on Chemotherapy of Tuberculosis, U.S. Veterans' Administration, Armed Forces, p. 17.

Marks, J. (1956). Acta tuberc. scand., 32, 63.

Medlar, E. M., Bernstein, S., and Steward, Dorothy M. (1952) Amer. Rev. Tuberc., 66, 36.

Stewart, Sheila M., Turnbull, F. W. A., and Macgregor, Agnes R. (1956). Tubercle (Lond.), 37, 388.

Sweany, H. C. (1941). Age Morphology of Primary Tubercles. Thomas, Springfield, Illinois.

Thomas, D. M. E. (1955). Med. Press, 233, 606.

Ware, P. F., Stauss, H. K., Dillon, R. J., and Tempel, C. W. (1956) Amer. Rev. Tuberc., 73, 165. 